\title{
Wireless Multichannel Acquisition of Neuropotentials
}

\author{
Mohsen Mollazadeh, Kartikeya Murari, \\ Helen Schwerdt, Xing Wang and Nitish Thakor \\ Department of Biomedical Engineering \\ Johns Hopkins University \\ Email: mohsenm@jhu.edu
}

\author{
Gert Cauwenberghs \\ Division of Biological Sciences \\ University of California San Diego \\ Email: gert@ucsd.edu
}

\begin{abstract}
Implantable brain-machine interfaces for disease diagnosis and motor prostheses control require low-power acquisition of neuropotentials spanning a wide range of amplitudes and frequencies. Here, we present a 16-channel VLSI neuropotential acquisition system with tunable gain and bandwidth, and variable rate digital transmission over an inductive link which further supplies power. The neuropotential interface chip is composed of an amplifier, incremental ADC and bit-serial readout circuitry. The front-end amplifier has a midband gain of $40 \mathrm{~dB}$ and offers NEF of less than 3 for all bandwidth settings. It also features adjustable low-frequency cut-off from 0.2 to $94 \mathrm{~Hz}$, and independent high-frequency cut-off from $140 \mathrm{~Hz}$ to $8.2 \mathrm{kHz}$. The Gm-C incremental $\Delta \Sigma$ ADC offers digital gain up to 4096 and 812 bits resolution. The interface circuit is powered by a telemetry chip which harvests power through inductive coupling from a $4 \mathrm{MHz}$ link, provides a $1 \mathrm{MHz}$ clock for ADC operation and transmits the bit-serial data of the neurpotential interface across $4 \mathrm{~cm}$ at up to $32 \mathrm{kbps}$ with a BER less than $10^{-5}$. Experimental EEG recordings using the neuropotential interface and wireless module are presented.
\end{abstract}

\section{INTRODUCTION}

Recent advances in the field of neural prosthesis have enabled decoding the multitude of electrical activity in the brain to understand neural mechanisms underlying movement [1], [2]. Neural signals occupy a wide range of signal frequencies and amplitudes, from $\mathrm{Hz}$ to $\mathrm{kHz}$ and from $\mu V$ to $\mathrm{mV}$. Action potentials, the electrical activity of a single neuron, occupy a bandwidth of $8 \mathrm{kHz}$ and have an amplitude of $500 \mu \mathrm{V}$. Recording spike activity from a population of neurons offer basic understanding underlying brain mechanism such as motor movements. However, microelectrodes need to be implanted in the brain to acquire these signals adding to the complexity associated with these recordings. On the other side of neural spectrum, electroencephalogram (EEG) is a representation of the electrical activity of the brain as recorded on the scalp. EEG signals are much weaker and slower than their spike counterparts, typically $50 \mu \mathrm{V}$ in amplitude and up to $100 \mathrm{~Hz}$ in frequency. EEG does not represent the exclusive activity of cortical regions below the electrodes because of volume conduction in the brain, which non-linearly adds single neuron activities. Inspite of their poor spatial resolution, a number of signal processing techniques have been developed to decode brain intent from EEG signals. These efforts demonstrate the possibility of control of a high degree of freedom prosthetic device using EEG signals.

Due to the noise, power, cost and footprint advantages of integrated systems, several VLSI systems have been developed for wired/wireless acquisition of neural signals [3]-[5]. For example, A wireless 100 channel system for recording single unit activity was reported by Harrison et al. [6]. The system uses a $2.64 \mathrm{MHz}$ inductive link to receive power and commands and telemeters the digitized data over a $433 \mathrm{MHz}$ FSK link. The amplifier has an input-referred noise of $5.1 \mu V_{r m s}$ while consuming $13.5 \mathrm{~mW}$ of power. Denison et al. [7] reported a neural amplifier for extraction of biomarkers which uses chopper stabilization to reduce input-referred noise of the amplifier. A $200 \mu \mathrm{W}, 8$ channel ASIC for ambulatory EEG monitoring was presented by Yazicioglu et al. [8]. Chae et al. [9] also reported a system with real time spike detection and an Ultra Wide Band (UWB) transmitter for acquisition of neural signals.

Here, we present a 16-channel integrated neuropotential interface chip with adjustable filtering, amplification and digitization covering the entire spectrum of neural signals. The system was powered by a telemetry chip previously designed in our lab [10] which harvests power from a $4 \mathrm{MHz}$ inductive link. The telemetry module was also used to transmit low frequency neural data (EEG, ECoG) over the same link back to the base station. The two chips were fabricated in a $0.5 \mu \mathrm{m}$ 2 poly, 3 metal CMOS process and the system maintained lownoise and low-power performance during wireless operation. Characterization results and in-vivo data are presented.

\section{SYSTEM ARCHITECTURE}

The functional block diagram of the wireless neural acquisition system is shown in Fig. 1. The interface chip has 16 parallel channels each comprising of a fully differential bandpass filtering two-stage voltage amplifier, a Gm-C incremental $\Delta \Sigma$ ADC, a decimating counter, and a daisy-chained parallelto-serial output register. The wireless chip supplies power and clock to and transmits data from the neuropotential interface chip using inductive coupling via a $4 \mathrm{MHz}$ link. The chip consists of a rectifier, two voltage regulators and circuits for clock recovery and data modulation [10]. The base station transmission coil voltage is driven by a high efficiency classE transmitter. On the receiving side, an off-chip coil feeds into a full wave rectifier, followed by a low pass filter and two separate regulators, to generate two $3.3 \mathrm{~V}$ supplies for the chip operation. Two supplies were used to decouple digital noise from the analog circuits. A clock recovery block extracts a $4 \mathrm{MHz}$ clock that is stepped down to $1 \mathrm{MHz}$ for the ADC operation. The NRZ format digitized data is transmitted back 

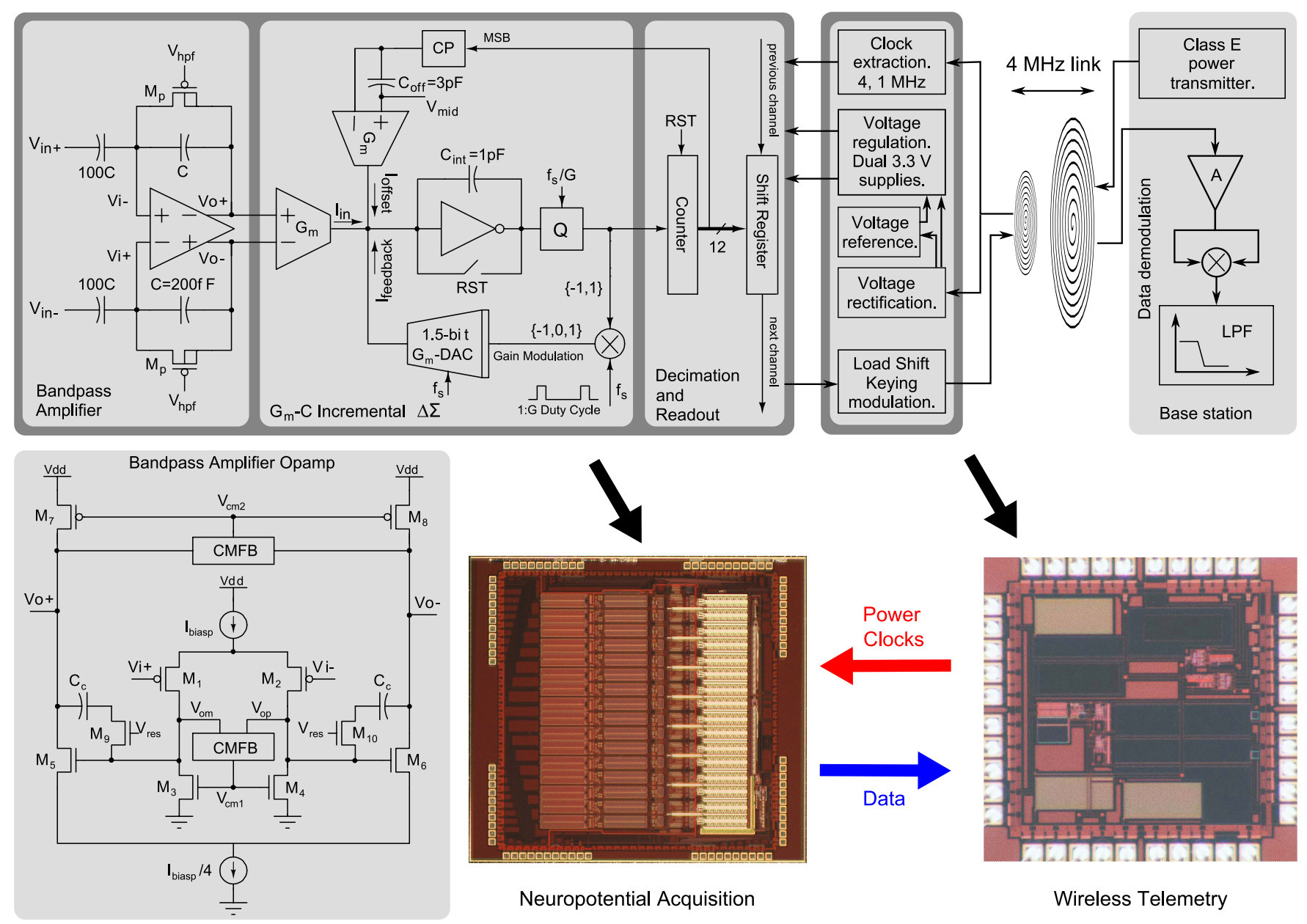

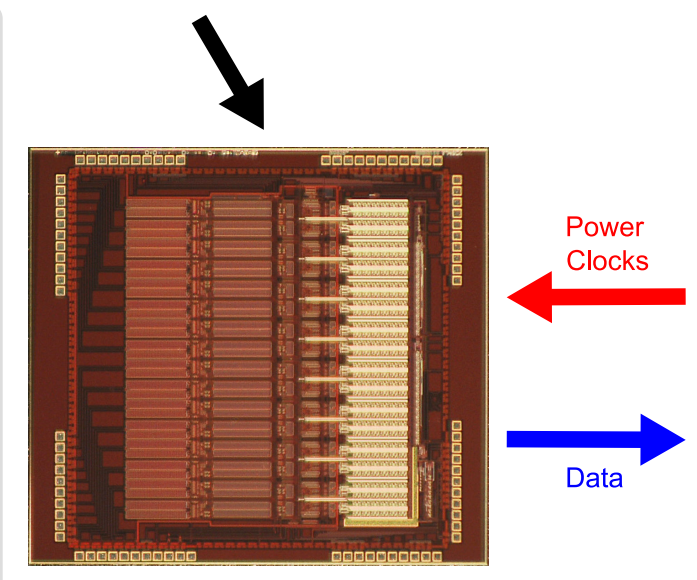

Neuropotential Acquisition

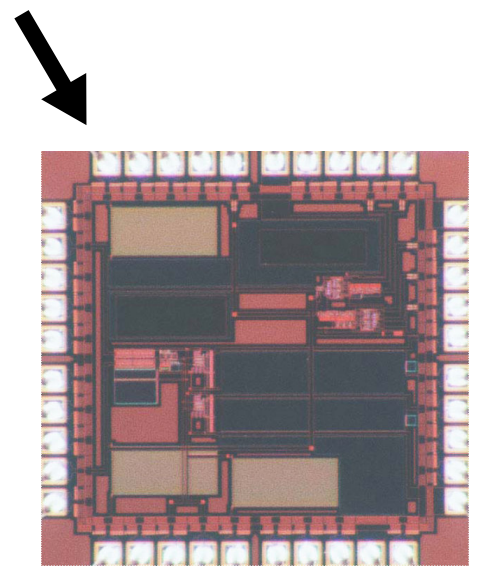

Wireless Telemetry

Fig. 1. Block diagram of wireless neuropotential acquisition system and the micrographs of the fabricated neuropotential acquisition and wireless telemetry chips. Circuit diagram of the bandpass amplifier in the interface chip is shown in the bottom left of the figure.

through the same link via Load Shift Key (LSK) modulation. At the base station, the received data is buffered, squared and low pass filtered to perform coherent demodulation.

In the interface chip, the amplifier midband gain is set to $40 \mathrm{~dB}$ by ratioed poly $1 /$ poly 2 capacitors $(100 C=20 \mathrm{pF}$ and $C=200 \mathrm{fF}$ ). The PMOS transistors $M_{p}$ in the feedback loop provide a large resistance in the $\mathrm{T} \Omega$ range for sub- $\mathrm{Hz}$ cutoff highpass filtering [4]. Tuning their gate bias, $V_{h p f}$, changes their resistance, which in turn adjusts the sub-hertz cutoff high pass filtering and $\mathrm{AC}$ coupling of the input. The low pass filter cutoff frequency can be tuned for various modalities of neural signals by changing the $g_{m}$ of the front-end amplifier which results in a change in its unity gain frequency.

The circuit implementing the fully differential two-stage voltage amplifier in the frontend amplifier is detailed in Fig. 1. Very wide $(\mathrm{W} / \mathrm{L}=216 \mu / 1.8 \mu)$ input PMOS transistors $M_{1}$ and $M_{2}$ permit weak inversion operation over a range of bias currents $I_{\text {biasp }}$ to maximize transconductance and hence minimize thermal noise. Large gate area sizing of $M_{1}$ and $M_{2}$ also partially mitigate the $1 / \mathrm{f}$ flicker noise. Likewise, second stage input transistors $M_{5}$ and $M_{6}$ were sized (W/L
$=18 \mu / 1.8 \mu)$ for weak inversion to minimize thermal noise, but at significantly lower bias currents. The attenuation of second stage input-referred noise by the first stage gain affords this lower current, and leads to significant savings in power consumption. The first and second stage bias currents are set in a constant ratio of 4:1 for an overall single-pole lowpass response of the two-stage amplifier. The unity gain frequency of the amplifier $g_{m} / 2 \pi C_{c}$ is set by the transconductance of the first stage $g_{m}$ proportional to the bias current $I_{b i a s p}$, and by $C_{c}=15 \mathrm{pF}$. Active resistors $M_{9}$ and $M_{10}$ were inserted to null the zero and non-dominant pole in the second-order response. Both stages have independent common mode feedback circuitry, CMFB (Fig. 1). The advantage over previous two-stage amplifier designs is superior noise efficiency approaching that of a single-stage single-ended amplifier, with reduced inputreferred noise and lower power contributed by the second stage and common-mode feedback.

The ADC was implemented using a continuous time Gm$\mathrm{C}$, incremental $\Delta \Sigma$ (Fig. 1). Each channel has a dedicated ADC for low clock speed operation reducing the power consumption. The ADC is configured in current-mode to in- 


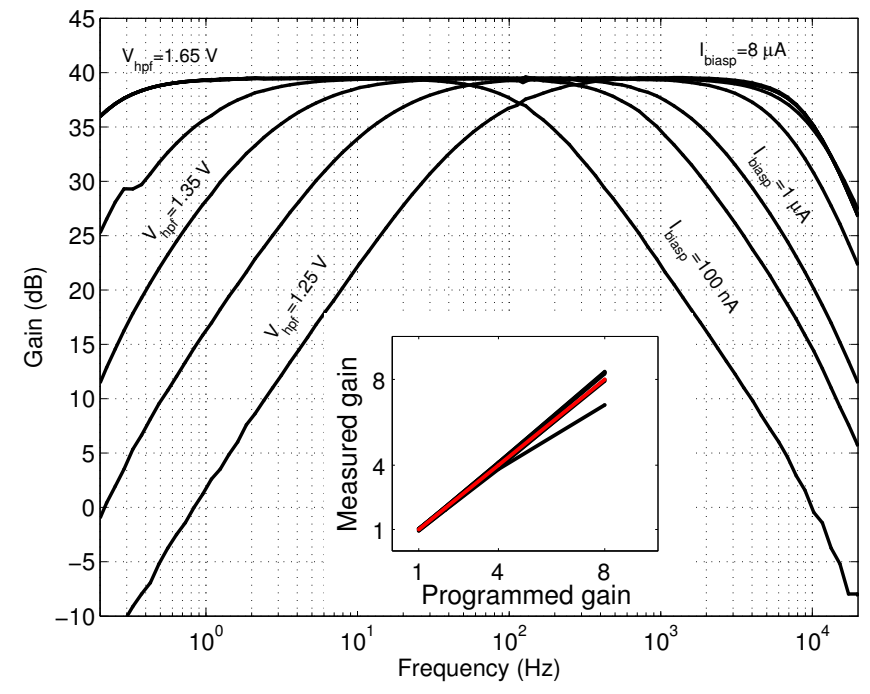

Fig. 2. Bandpass amplifier responses for lowpass current bias $I_{\text {biasp }}$ between $100 \mathrm{nA}$ and $8 \mu \mathrm{A}$, and for highpass voltage bias $V_{h p f}$ between $1.25 \mathrm{~V}$ and $1.65 \mathrm{~V}$. The inset shows the normalized measured gain versus programmed gain for multiple channels. The red trace is the ideal unity slope line.

corporate a time modulation feedback which allows a digitally programmable gain between 1 and 4096. The structure also allows configuration of the ADC resolution [11]. In order to remove any offset in the digitized output caused by mismatch in the ADCs, a charge pump based circuit adaptively adds or subtracts a small current from the OTA based on the output of the ADC. The digital output is decimated using a first order comb filter and read out from a parallel-in serial-out shift register.

\section{EXPERIMENTAL RESULTS}

The neural interface and wireless power harvesting and data telemetry blocks were fabricated separately in a $0.5 \mu \mathrm{m}$ 3M2P CMOS process through the MOSIS foundry service. The 16 channels of neural interface occupy $3 \mathrm{~mm} \times 3 \mathrm{~mm}$ of silicon area and consume $1.8 \mathrm{~mW}$ of power at the maximum bandwidth and speed $(8.2 \mathrm{kHz}$ and $16 \mathrm{kS} / \mathrm{s})$. Each block of the system was characterized independently. For characterizing the system and in vivo recordings, the digital output (or demodulated data) of the chip was acquired using a DAQ card (National Instruments, Austin, TX) and read into a computer.

\section{A. Neuropotential Interface}

Measured gain and bandwidth of the closed-loop bandpass amplifier are shown in Fig. 2. The midband gain was $39.6 \mathrm{~dB}$. The lower cutoff frequency could be varied between $0.2 \mathrm{~Hz}$ to $94 \mathrm{~Hz}$ by tuning $V_{h p f}$ from 3.3 to $1.25 \mathrm{~V}$. The higher cutoff frequency was adjustible from $140 \mathrm{~Hz}$ to $8.2 \mathrm{kHz}$ by tuning $I_{\text {biasp }}$ from 0.1 to $8 \mu \mathrm{A}$. The inset figure plots measured vs programmed gain of all channels showing mismatch in the system channels and the deviation from the digitally programmed gain in the ADC stage. The gain was set to 1, 2, 4 and 8 and the input sine wave amplitude was halved each time. The ADC resolution was changed from 12 to 9 bits to

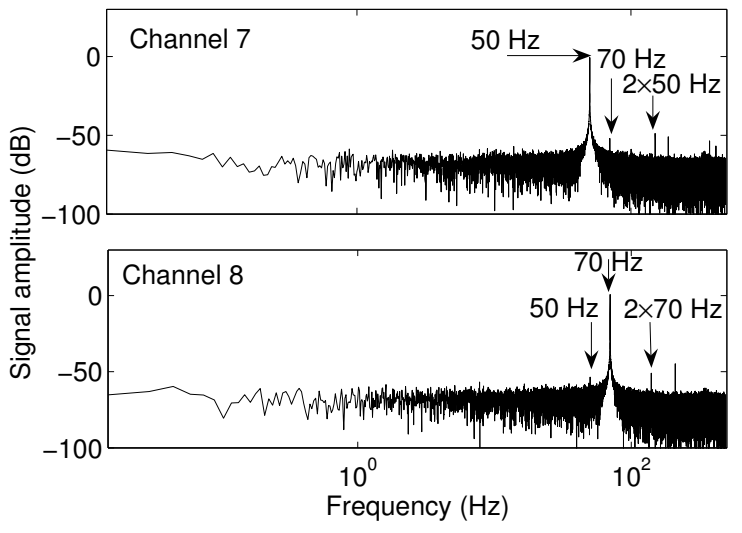

Fig. 3. Normalized power spectra of the recorded digital output for two adjacent channels. $1 \mathrm{mV} 50 \mathrm{~Hz}$ and $70 \mathrm{~Hz}$ sine waves were presented to the channel 7 and 8 of the interface respectively.

maintain the sampling rate. The red trace is the ideal unity slope line.

The amplifier's CMRR and PSRR were larger than $76 \mathrm{~dB}$ for inputs between $1 \mathrm{~Hz}$ and $10 \mathrm{kHz}$ at an electrode offset of $50 \mathrm{mV}$. The amplifier THD was below $1 \%$ for inputs within $9.4 m V_{p-p}$. For the $8.2 \mathrm{kHz}$ bandwidth $\left(I_{\text {biasp }}=8 \mu \mathrm{A}\right.$ and $\left.V_{h p f}=3.3 \mathrm{~V}\right)$, the amplifier had a thermal noise level of $18 \mathrm{nV} / \sqrt{\mathrm{Hz}}$ and a $1 / \mathrm{f}$ noise corner at $1 \mathrm{kHz}$. Integration of the PSD for this bandwidth yielded an input-referred noise of $1.94 \mu V_{r m s}$ and a noise efficiency factor (NEF) of 2.9. Decreasing the amplifier bias to the lowest level $\left(I_{\text {biasp }}=\right.$ $100 \mathrm{nA}$ ) resulted in higher thermal noise level but lower 1/f corner frequency, with an input-referred noise of $1.65 \mu V_{r m s}$ and an NEF of 3.2.

Fig. 3 shows the power spectra of the recorded digital output of two adjacent channels. The data indicate a THD of $0.5 \%$, and a digital output noise of 1.1 LSB and more than $55 \mathrm{~dB}$ crosstalk suppression. Lower quantization noise levels can be attained by higher gain setting for smaller signal amplitudes.

\section{B. Wireless Power Harvesting and Telemetry}

A transponder coil of $1 \mathrm{~cm}$ radius was used to receive power and clock using inductive coupling at $4 \mathrm{MHz}$ from a reader coil of diameter $2.5 \mathrm{~cm}$. Fig. 4 shows the $3.3 \mathrm{~V}$ generated supply, $32 \mathrm{kHz}$ data clock (generated from $1 \mathrm{MHz}$ recovered clock), transmitted neural data and demodulated neural data at the base station. Stable operation of the circuit was achieved with up to $4 \mathrm{~cm}$ separation between the coils. Data transmission was also tested up to $32 \mathrm{kbps}$ resulting in a bit error rate (BER) less than $10^{-5}$. With wireless operation of the system, the channel noise increased to $2.1 \mathrm{LSB}$ due to increased noise on the power supplies.

\section{In-vivo Recordings}

Although the neuropotential interface chip is capable of recording spike signals $(8.2 \mathrm{kHz})$, the telemetry system limits the data transfer rate since the same link is used for transmitting data as well as power. For the purpose of this report, 
TABLE I
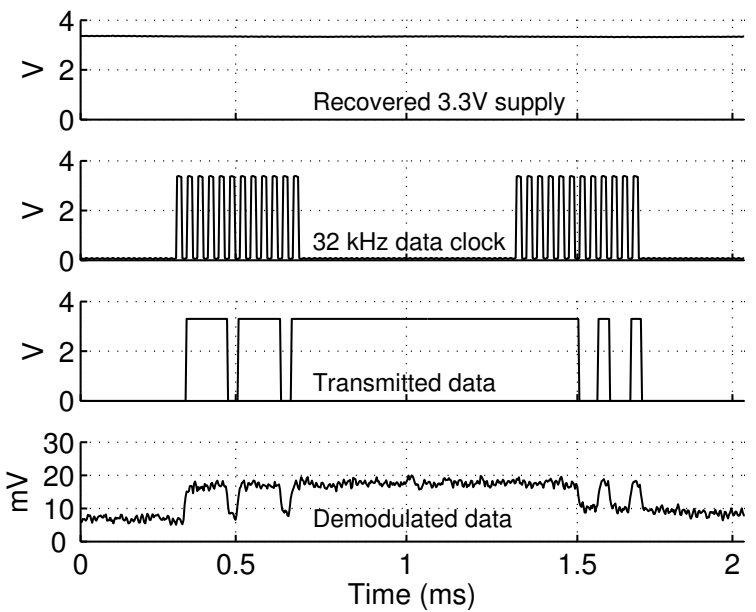

Fig. 4. RF operation: $3.3 \mathrm{~V}$ generated supply, $32 \mathrm{kHz}$ data clock (generated from the $1 \mathrm{MHz}$ recovered clock), transmitted data and demodulated data at the base station.

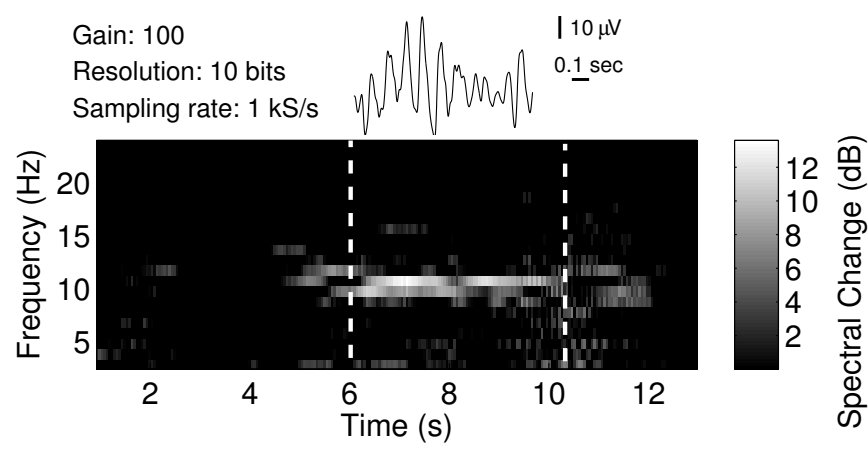

Fig. 5. Time frequency plot of the recorded EEG waveform from occipital lobe of a human subject. Dotted line shows closing and opening of the eye. The recorded EEG waveform when the eyes are closed is shown on top.

we will present EEG recordings with wireless powering and telemetry. EEG recordings were performed on a male human subject fitted with a 20-electrode cap with gel-based electrodes (Electro-Cap, Eaton, OH). The O1 electrode located above the occipital lobe was connected to the interface circuit and the subject was asked to periodically open and close his eyes for $4 \mathrm{~s}$ intervals. Fig. 5 shows the time frequency plot of the recorded waveform under wireless operation. Dotted lines denote the closing and opening of eye. As it can be seen, the power in the $11 \mathrm{~Hz}$ band ( $\alpha$ activity) increases during eye closure.

\section{CONCLUSIONS}

We have presented an integrated multichannel acquisition system for recording neural signals. Tunable filters were embedded in the amplifier front-end stage in order to selectively amplify the signal of interest. The amplifier's input-referred noise was below $2 \mu V_{r m s}$, consistently providing low-noise performance. The configurable gain and resolution setting in the ADC stage allows the optimum quantization based on the dynamic range and frequency content of the acquired signal.
PERFORMANCE SUMMARY OF THE SYSTEM

\begin{tabular}{|l|l|l|l|}
\hline \multirow{4}{*}{ Amplifier } & Gain & 39.6 & $\mathrm{~dB}$ \\
& Lowpass cutoff & $0.140-8.2$ & $\mathrm{kHz}$ \\
& Highpass cutoff & $0.2-94$ & $\mathrm{~Hz}$ \\
& THD, $v_{\text {in }} \leq 9.4 m V_{p p}$ & $<1 \%$ & \\
& Noise Efficiency Factor & $<3$ & \\
\hline \multirow{3}{*}{ Channel } & Programmable gain & $1-4096$ & \\
& Sampling rate & $<16$ & $\mathrm{kS} / \mathrm{s}$ \\
& Noise & $<1.1$ & $\mathrm{LSB}$ \\
& Current draw & $<34$ & $\mu A$ \\
\hline RF operation & Harvested power (dual supplies) & 3.3 & $\mathrm{~V}$ \\
over 4 cm & Channel noise & $<2.1$ & $\mathrm{LSB}$ \\
\& 32 kbps & Bit error rate & $<10^{-5}$ & \\
\hline \multirow{2}{*}{ Chip } & Power (maximum BW \& speed) & 1.8 & $\mathrm{~mW}$ \\
& Gain mismatch & $<5 \%$ & \\
& Crosstalk suppression & $<-55$ & $\mathrm{~dB}$ \\
\hline
\end{tabular}

The wireless power harvesting module was able to provide the power and clock for interface operation at distances up to $4 \mathrm{~cm}$ and transmit data up to $32 \mathrm{kbps}$ rate with negligible BER. RF operation increased the input-referred noise of the system from 1.1 to 2.1 LSB. The system was used to wirelessly acquire EEG from a human subject. The performance of the system is summarized in Table I.

\section{ACKNOWLEDGEMENT}

This work was supported by NIH MH062444-065296 and NIH/NIA 1R01AG029681 grants.

\section{REFERENCES}

[1] L. R. Hochberg et al., "Neuronal ensemble control of prosthetic devices by a human with tetraplegia," Nature, vol. 442, no. 13, pp. 164-171, 2006.

[2] E. C. Leuthardt, G. Schalk, J. R. Wolpaw, G. J. Ojemann, and D. W. Moran, "A brain-computer interface using electrocorticographic signals in humans," J. Neural Eng., vol. 1, no. 2, pp. 63-71, 2004.

[3] P. Mohseni and K. Najafi, "A fully integrated neural recording amplifier with dc input stabilization," IEEE Trans. Biomed. Eng., vol. 51, no. 5, pp. 832-837, 2004.

[4] R. Harrison and C. Charles, "A low-power low-noise CMOS amplifier for neural recording applications," IEEE J. Solid-State Circuits, vol. 38, no. 6, pp. 958-965, 2003.

[5] Y. Perelman and R. Ginosar, "An integrated system for multichannel neuronal recording with spike/LFP separation, integrated A/D conversion and threshold detection," IEEE Trans. Biomed. Eng., vol. 54, no. 1, pp. 130-137, 2007.

[6] R. R. Harrison et al., "A low-power integrated circuit for a wireless 100 electrode neural recording system," IEEE J. Solid-State Circuits, vol. 42, no. 1 , pp. 123-133, 2007.

[7] T. Denison, W. Santa, R. Jensen, D. Carlson, G. Molnar, and A.-T. Avestruz, "An $8 \mu \mathrm{W}$ heterodyning chopper amplifier for direct extraction of 2uvrms neuronal biomarkers," Solid-State Circuits Conference, 2008. ISSCC 2008. Digest of Technical Papers. IEEE International, pp. 162 603, Feb. 2008.

[8] R. F. Yazicioglu, P. Merken, R. Puers, and C. Van Hoof, "A $200 \mu \mathrm{W}$ eight-channel acquisition asic for ambulatory eeg systems," ISSCC 2008. Digest of Technical Papers, pp. 164-603, Feb. 2008.

[9] M. Chae et al., "A 128 -channel $6 \mathrm{~mW}$ wireless neural recording ic with on-the-fly spike sorting and uwb tansmitter," ISSCC 2008. Digest of Technical Papers, pp. 146-603, Feb. 2008.

[10] C. Sauer, M. Stanacevic, G. Cauwenberghs, and N. Thakor, "Power harvesting and telemetry in CMOS for implanted devices," IEEE Trans. Circuits Sys. I: Regular Papers, vol. 52, no. 12, pp. 2605-2613, 2005.

[11] M. Stanacevic, K. Murari, A. Rege, G. Cauwenberghs, and N. Thakor, "VLSI potentiostat array with oversampling gain modulation for widerange neurotransmitter sensing," IEEE Trans. Biomed. Circ. Sys., vol. 1, no. 1, pp. 63-72, 2007. 